\title{
Phase mixing of Alfvén waves propagating in non-reflective magnetic plasma configurations
}

\author{
Michael S. Ruderman ${ }^{1,2}$ and Nikolai S. Petrukhin ${ }^{3}$ \\ ${ }^{1}$ School of Mathematics and Statistics (SoMaS), The University of Sheffield, Hicks Building, Hounsfield Road, Sheffield S3 7RH, \\ UK \\ e-mail: m.s.ruderman@sheffield.ac.uk \\ 2 Space Research Institute (IKI), Russian Academy of Sciences, Moscow 117342, Russia \\ ${ }^{3}$ National Research University Higher School of Economics, Moscow 101000, Russia
}

Received 13 October 2016 / Accepted 18 January 2017

\begin{abstract}
The ability of phase mixing to provide efficient damping of Alfvén waves even in weakly dissipative plasmas made it a popular mechanism for explaining the solar coronal heating. Initially it was studied in the equilibrium configurations with the straight magnetic field lines and the Alfvén speed only varying in the direction perpendicular to the magnetic field. Later the analysis of the Alfvén wave phase mixing was extended in various directions. In particular it was studied in two-dimensional planar magnetic plasma equilibria. Analytical investigation was carried out under the assumption that the wavelength is much smaller than the characteristic scale of the background quantity variation. This assumption enabled using the Wentzel, Kramers, and Brillouin (WKB) method. When it is not satisfied the study was only carried out numerically. In general, even the wave propagation in a one-dimensional inhomogeneous equilibrium can be only studied numerically. However there is one important exception, so-called non-reflective equilibria. In these equilibria the wave equation with the variable phase speed reduces to the Klein-Gordon equation with constant coefficients. In this paper we apply the theory of non-reflective wave propagation to studying the Alfvén wave phase mixing in two-dimensional planar magnetic plasma equilibria. Using curvilinear coordinates we reduce the equation describing the Alfvén wave phase mixing to the equation that becomes a one-dimensional wave equation in the absence of dissipation. This equation is further reduced to the equation which is the one-dimensional Klein-Gordon equation in the absence of dissipation. Then we show that this equation has constant coefficients when a particular relation between the plasma density and magnetic field magnitude is satisfied. Using the derived KleinGordon-type equation we study the phase mixing in various non-reflective equilibria. We emphasise that our analysis is valid even when the wavelength is comparable with the characteristic scale of the background quantity variation. In particular, we study the Alfvén wave damping due to phase mixing in an equilibrium with constant plasma density and exponentially divergent magnetic field lines. We confirm the result previously obtained in the WKB approximation that there is enhanced Alfvén wave damping in this equilibrium with the damping length proportional to $\ln (\mathrm{Re})$, where $\mathrm{Re}$ is the Reynolds number. Our theoretical results are applied to heating of coronal plumes. We show that, in spite of enhanced damping, Alfvén waves with periods of the order of one minute can be efficiently damped in the lower corona, at the height about $200 \mathrm{Mm}$, only if the shear viscosity is increased by about 6 orders of magnitude in comparison with its value given by the classical plasma theory. We believe that such increase of the shear viscosity can be provided by the turbulence.
\end{abstract}

Key words. hydrodynamics - magnetohydrodynamics (MHD) - plasmas - waves - Sun: corona - Sun: oscillations

\section{Introduction}

Over the last few decades the problem of solar coronal heating has been one of the hot topics in solar physics. One popular mechanism for heating the coronal plasma is by Alfvén waves. A remarkable property of Alfvén waves is that they can easily transform the energy from the lower part of the solar atmosphere to the corona. However, for typical coronal conditions, Alfvén waves can propagate in a homogeneous or even vertically stratified coronal plasma practically without damping because they do not perturb the plasma density, while the damping due to shear viscosity and resistivity is extremely weak.

Heyvaerts \& Priest (1983) suggested a mechanism that can enormously enhance the Alfvén wave damping in weakly dissipative plasmas. This mechanism is called phase mixing. It works when the Alfvén speed varies in the direction perpendicular to the direction of wave propagation. This variation of the Alfvén speed makes the waves propagating along neighbouring magnetic field lines more and more our of phase. This causes large gradients in the direction perpendicular to the wave propagation direction to build up, which leads to strong wave damping. As a result, the damping length is proportional to $\mathrm{Re}^{1 / 3}$ rather than $\mathrm{Re}$ as it is in a homogeneous plasma, where $\mathrm{Re}$ is either the viscous or resistive Reynolds number. The possibility of efficiently damping Alfvén waves in weakly dissipative plasmas made phase mixing a popular mechanism for explaining the heating of open magnetic structures in the solar corona. One recent example is the article by Tsiklauri (2016) where the effect of flow on the dissipation of Alfvén waves due to phase mixing is studied.

The initial model suggested by Heyvaerts \& Priest (1983) was developed in various directions. In particular, Hood et al. (1997a,b) found analytical, self-similar solutions describing phase mixing of Alfvén waves in both open (coronal holes) and closed (coronal loops) magnetic configurations. Hood et al. (2002) studied the damping due to phase mixing of Alfvén wave 
pulses propagating in the equilibrium with the straight magnetic field lines and density only varying in the direction orthogonal to the magnetic field. The main result that they obtained is that in this case the Alfvén wave amplitude does not decay exponentially. Instead, its damping is described by a power law. Hood et al. (2002) considered the propagation of either a single pulse or a pair of pulses with the opposite polarity. Hood et al. (2005) extended this analysis and studied the propagation of a train of pulses. When there are many pulses in the train, they found that the amplitude decay of the leading and trailing pulses is still described by a power law, while the amplitudes of internal pulses decay exponentially.

Although phase mixing strongly enhances the Alfvén wave damping, it is still not strong enough to provide damping in the lower corona. This prompted researchers to look for new effects that can further enhance the wave damping. Malara et al. (1996) showed that compressible perturbation can be generated during the phase mixing of Alfvén waves. The perturbation is subjected to non-linear steepening which results in the appearance of shocks. This idea was further developed by Nakariakov et al. (1997, 1998), Botha et al. (2000), Tsiklauri et al. (2001, 2002, 2003), and Tsiklauri \& Nakariakov (2002).

Initially the Alfvén wave phase mixing was only studied in magnetic plasma configurations with the straight magnetic field and the Alfvén speed only varying in the direction perpendicular to the magnetic field. However, in real magnetic plasma configurations in the solar atmosphere the magnetic field lines are very often curved, and the Alfvén speed depends on at least two spatial coordinates. Ruderman et al. (1998) studied the Alfvén wave mixing in two-dimensional magnetic plasma configurations under the assumption that the curvature of the magnetic field lines and the characteristic scale of the Alfvén speed variation along the magnetic field lines are both much larger than the wavelength. The solution was obtained using the Wentzel, Kramers, and Brillouin (WKB) method. In particular, Ruderman et al. (1998) showed that the exponential growth of the Alfvén speed with the height in a magnetic plasma configuration with the vertical magnetic field suppresses the wave damping due to phase mixing and thus leads to the increase in the damping length. Later Ruderman et al. (1999) extended the analysis by Ruderman et al. (1998) to axisymmetric magnetic plasma configurations with purely poloidal equilibrium magnetic fields in the presence of plasma flow.

The results obtained by Ruderman et al. (1998) in the WKB approximation were confirmed by De Moortel et al. (1999), who studied numerically the damping of Alfvén waves due to phase mixing in a stratified atmosphere with straight magnetic field lines. Their numerical analysis is valid for waves with the wavelength comparable to the atmospheric scale height De Moortel et al. (2000) extended this analysis to a stratified atmosphere with the radially divergent magnetic field lines.

The most interesting result obtained by Ruderman et al. (1998) was that strong divergence of the magnetic field lines can substantially enhance the efficiency of the Alfvén wave damping due to phase mixing. In particular it was shown that while the damping length in an equilibrium with the straight magnetic field lines and the Alfvén speed only varying in the direction orthogonal to the magnetic field direction is proportional to $\mathrm{Re}^{1 / 3}$, in an equilibrium with the exponentially divergent magnetic field lines it is proportional to $\ln (\mathrm{Re})$. This result was confirmed by Smith et al. (2007) who studied the problem of the Alfvén wave damping due to phase mixing in an equilibrium with the exponentially divergent magnetic field lines both analytically and numerically.
The analytical studies by both Ruderman et al. (1998) and Smith et al. (2007) were carried out under the assumption that the wavelength is much smaller than the characteristic scale of the equilibrium quantity variation. This assumption enabled using the WKB method. When the wavelength is comparable with the equilibrium characteristic scale then, in general, the study of the Alfvén phase mixing is only possible numerically. However, there is one exception: it is possible in equilibria where Alfvén waves propagate without reflection.

Non-reflective wave propagation was studied in various branches of sciences. It was studied in plasma physics (Ginzburg 1970), oceanography (Brekhovskih 1980; Didenkulova et al. 2008; Grimshaw et al. 2010), acoustics (Ibragimov \& Rudenko 2004), and atmospheric science (Petrukhin et al. 2011). Recently the theory of non-reflective wave propagation has been applied to solar physics. Petrukhin et al. (2012) studied non-reflective vertical propagation of acoustic waves in the solar atmosphere. Cally (2012) investigated non-reflective propagation of Alfvén waves. Ruderman et al. (2013) and Petrukhin et al. (2015) studied non-reflective propagation of kink waves along thin magnetic flux tubes.

This article aims to study the phase mixing of propagating Alfvén waves in non-reflective equilibria. It is organised as follows. In the next section we formulate the problem and derive the equation governing the velocity perturbation in shear Alfvén waves. In Sect. 3 we introduce curvilinear coordinates with one set of coordinate lines coinciding with the magnetic field lines and the other coinciding with the level lines of potential of the equilibrium magnetic field. We then transform the governing equation for the velocity to these coordinates. In Sect. 4 we study the non-reflective propagation of Alfvén waves. In Sect. 5 we develop the general theory of Alfvén wave damping due to phase mixing in equilibria where these waves propagate without reflection. In Sect. 6 we study the wave damping in equilibria with straight magnetic field lines. In Sect. 7 the wave damping is studied in an equilibrium with the divergent magnetic field lines. The results are applied to the Alfvén wave damping in coronal plumes. Section 8 presents a summary of the results and our conclusions.

\section{Equilibrium state and governing equations}

We consider a two-dimensional equilibrium state where all equilibrium quantities depend on $x$ and $z$ in Cartesian coordinates $x, y, z$ with the $z$-axis vertical. The $y$-component of the equilibrium magnetic field is zero. The plasma beta in the solar corona is very low, which implies that the equilibrium magnetic field must be force-free. A force-free two-dimensional magnetic field is always potential, hence the equilibrium magnetic field $\boldsymbol{B}=\left(B_{x}, 0, B_{z}\right)$ can be expressed in terms of magnetic potential $\phi$. It can be also expressed in terms of the magnetic flux function $\psi$. As a result, we have

$$
\frac{B_{x}}{B_{0}}=\frac{\partial \phi}{\partial x}=-\frac{\partial \psi}{\partial z}, \quad \frac{B_{z}}{B_{0}}=\frac{\partial \phi}{\partial z}=\frac{\partial \psi}{\partial x},
$$

where $B_{0}$ is a constant equal to the characteristic value of the magnetic field. Below we use $\phi$ and $\psi$ as new curvilinear coordinates in the $x z$-plane. It follows from Eq. (1) that $\nabla \phi \cdot \nabla \psi=0$. This means that the curvilinear coordinate system $(\phi, \psi)$ is orthogonal. The $\phi$ coordinate lines coincide with the magnetic field lines, while the $\psi$ coordinate lines are orthogonal to the magnetic field lines. We consider the plasma density $\rho$ as a free function. If this function is given, then the plasma pressure and temperature 
are determined by the projection of the equilibrium equation on the magnetic field direction and the ideal gas law.

Below we study Alfvén waves where only the $y$-components of the velocity and magnetic field, $u$ and $b$, are non-zero, while all other quantities remain unperturbed. To describe these waves we use the $y$-components of the momentum and induction equation. The only dissipative process that we take into account is shear viscosity, while we neglect resistivity. Some authors (e.g. Heyvaerts \& Priest 1983) took both shear viscosity and resistivity into account. As a result, they obtained that the term describing dissipation in the equation governing the Alfvén wave propagation is multiplied by the sum of the kinematic viscosity and magnetic diffusion. In the corona these quantities are of the same order. This implies that neglecting one of them can only reduce the efficiency of wave damping by factor of the order of unity. On the other hand, neglecting resistivity simplifies the analysis because it enables the magnetic field perturbation to be easily eliminated from the governing equations. The linearised governing equations are

$$
\begin{aligned}
& \rho \frac{\partial u}{\partial t}=\frac{1}{\mu_{0}} \boldsymbol{B} \cdot \nabla b+\frac{\partial}{\partial x}\left(\rho v \frac{\partial u}{\partial x}\right)+\frac{\partial}{\partial z}\left(\rho v \frac{\partial u}{\partial z}\right), \\
& \frac{\partial b}{\partial t}=\boldsymbol{B} \cdot \nabla u,
\end{aligned}
$$

where $\mu_{0}$ is the magnetic permeability of free space and $v$ the kinematic viscosity. Eliminating $b$ from these equations we obtain the equation for $u$ :

$$
\begin{aligned}
\rho \frac{\partial^{2} u}{\partial t^{2}}= & \frac{1}{\mu_{0}} \boldsymbol{B} \cdot \nabla(\boldsymbol{B} \cdot \nabla u) \\
& +\frac{\partial}{\partial t}\left[\frac{\partial}{\partial x}\left(\rho v \frac{\partial u}{\partial x}\right)+\frac{\partial}{\partial z}\left(\rho v \frac{\partial u}{\partial z}\right)\right] .
\end{aligned}
$$

\section{Introducing curvilinear coordinates}

We now make the variable substitution and use $\phi$ and $\psi$ as the independent variables. Then we have the following expressions for the partial derivatives with respect to $x$ and $z$ :

$$
\frac{\partial}{\partial x}=\frac{B_{x}}{B_{0}} \frac{\partial}{\partial \phi}+\frac{B_{z}}{B_{0}} \frac{\partial}{\partial \psi}, \quad \frac{\partial}{\partial z}=\frac{B_{z}}{B_{0}} \frac{\partial}{\partial \phi}-\frac{B_{x}}{B_{0}} \frac{\partial}{\partial \psi} .
$$

It follows from these results that

$$
\boldsymbol{B} \cdot \nabla=\frac{B^{2}}{B_{0}} \frac{\partial}{\partial \phi} .
$$

Using Eqs. (5) and (6) we transform Eq. (4) to

$$
\begin{aligned}
& \frac{\partial^{2} u}{\partial t^{2}}-V_{\mathrm{A}}^{2} \frac{\partial}{\partial \phi} \frac{B^{2}}{B_{0}^{2}} \frac{\partial u}{\partial \phi}= \\
& \frac{1}{\rho} \frac{\partial}{\partial t}\left[\left(\frac{B_{x}}{B_{0}} \frac{\partial}{\partial \phi}+\frac{B_{z}}{B_{0}} \frac{\partial}{\partial \psi}\right) \rho v\left(\frac{B_{x}}{B_{0}} \frac{\partial u}{\partial \phi}+\frac{B_{z}}{B_{0}} \frac{\partial u}{\partial \psi}\right)\right. \\
& \left.\quad+\left(\frac{B_{z}}{B_{0}} \frac{\partial}{\partial \phi}-\frac{B_{x}}{B_{0}} \frac{\partial}{\partial \psi}\right) \rho v\left(\frac{B_{z}}{B_{0}} \frac{\partial u}{\partial \phi}-\frac{B_{x}}{B_{0}} \frac{\partial u}{\partial \psi}\right)\right],
\end{aligned}
$$

where

$$
V_{\mathrm{A}}^{2}=\frac{B^{2}}{\mu_{0} \rho} \text {. }
$$

Below we assume that the viscosity is small and it is only important when small spatial scales in the direction orthogonal to the magnetic field lines are formed due to phase mixing. In this case the second derivative of $u$ with respect to $\psi$ strongly dominates all other terms on the right-hand side of Eq. (7). As a result we can rewrite Eq. (4) in the approximate form

$$
\frac{\partial^{2} u}{\partial t^{2}}-V_{\mathrm{A}}^{2} \frac{\partial}{\partial \phi} \frac{B^{2}}{B_{0}^{2}} \frac{\partial u}{\partial \phi}=v \frac{B^{2}}{B_{0}^{2}} \frac{\partial^{3} u}{\partial t \partial \psi^{2}} .
$$

This is the main equation that is used below to study the damping of Alfvén waves due to phase mixing.

\section{Non-reflective propagation of Alfvén waves}

In this section we describe how Eq. (9) can be reduced to an equation with constant coefficients. The reduction of the wave equation with the variable phase speed to the Klein-Gordon equation with constant coefficients is described in many articles (see, e.g. Ruderman et al. 2013). However, Eq. (9) is slightly different from the standard wave equation and, as a result, its reduction to an equation with constant coefficients is also slightly different from the standard reduction of wave equation to the Klein-Gordon equation.

We search for the solution to Eq. (9) in the form

$u(t, \phi, \psi)=A(\phi, \psi) \Phi(t, h(\phi, \psi), \psi)$,

where $A(\phi, \psi)$ and $\Phi(t, h(\phi, \psi), \psi)$ are the functions to be determined. Substituting this expression in Eq. (9) we obtain

$$
\begin{aligned}
& \frac{\partial^{2} \Phi}{\partial t^{2}}-V_{\mathrm{A}}^{2} \frac{B^{2}}{B_{0}^{2}}\left(\frac{\partial h}{\partial \phi}\right)^{2} \frac{\partial^{2} \Phi}{\partial h^{2}}-\frac{V_{\mathrm{A}}^{2}}{A}\left[\frac{B^{2}}{B_{0}^{2}} \frac{\partial A}{\partial \phi} \frac{\partial h}{\partial \phi}\right. \\
& \left.+\frac{\partial}{\partial \phi}\left(\frac{A B^{2}}{B_{0}^{2}} \frac{\partial h}{\partial \phi}\right)\right] \frac{\partial \Phi}{\partial h}-V_{\mathrm{A}}^{2} \frac{\Phi}{A} \frac{\partial}{\partial \phi}\left(\frac{B^{2}}{B_{0}^{2}} \frac{\partial A}{\partial \phi}\right)= \\
& \frac{v B^{2}}{B_{0}^{2}} \frac{\partial}{\partial t}\left[\frac{1}{A} \frac{\partial^{2}(A \Phi)}{\partial \psi^{2}}+\frac{2}{A} \frac{\partial h}{\partial \psi} \frac{\partial}{\partial \psi}\left(A \frac{\partial \Phi}{\partial h}\right)\right. \\
& \left.+\frac{\partial^{2} h}{\partial \psi^{2}} \frac{\partial \Phi}{\partial h}+\left(\frac{\partial h}{\partial \psi}\right)^{2} \frac{\partial^{2} \Phi}{\partial h^{2}}\right] .
\end{aligned}
$$

Now we impose the condition that the coefficient at the second derivative of $\Phi$ with respect to $h$ on the left-hand side of this equation is equal to $V_{0}^{2}$, and the coefficient at the first derivative is zero, where $V_{0}$ is the characteristic value of the Alfvén speed:

$\frac{B^{2} V_{\mathrm{A}}^{2}}{B_{0}^{2} V_{0}^{2}}\left(\frac{\partial h}{\partial \phi}\right)^{2}=1, \frac{B^{2}}{B_{0}^{2}} \frac{\partial A}{\partial \phi} \frac{\partial h}{\partial \phi}+\frac{\partial}{\partial \phi}\left(\frac{A B^{2}}{B_{0}^{2}} \frac{\partial h}{\partial \phi}\right)=0$.

It follows from these equations that

$h=B_{0} V_{0} \int_{\phi_{1}}^{\phi} \frac{\mathrm{d} \phi^{\prime}}{B V_{\mathrm{A}}}, \quad A=\rho^{-1 / 4}$,

where $\phi_{1}$ is a function of $\psi$ that, at present, we do not specify. When deriving the expression for $A$ we have taken into account that $A$ is defined with an accuracy up to a multiplicative constant. If we now take $v=0$, then Eq. (11) reduces to the Klein-Gordon equation

$\frac{\partial^{2} \Phi}{\partial t^{2}}-V_{0}^{2} \frac{\partial^{2} \Phi}{\partial h^{2}}=V_{\mathrm{A}}^{2} \frac{\Phi}{A} \frac{\partial}{\partial \phi}\left(\frac{B^{2}}{B_{0}^{2}} \frac{\partial A}{\partial \phi}\right)$.

In general, this is an equation with one variable coefficient. This causes reflection of a wave driven at a level $\phi=$ const. and propagating in the positive $\phi$-direction. It can only propagate without 
reflection if the coefficient at $\Phi$ in Eq. (14) is constant. However, we must keep in mind that $\psi$ is present in Eq. (14) as a parameter, so this constant can depend on $\psi$. Hence, we impose the condition that the coefficient at $\Phi$ in Eqs. (11) and (14) only depends on $\psi$,

$\frac{V_{\mathrm{A}}^{2}}{A} \frac{\partial}{\partial \phi}\left(\frac{B^{2}}{B_{0}^{2}} \frac{\partial A}{\partial \phi}\right)=\frac{\sigma(\psi)}{\mu_{0} B_{0}^{2}}$,

where $\sigma(\psi)$ is an arbitrary function.

Finally, we recall that the viscosity is only important when there is small spatial scale in the $\psi$ direction. Below we study the initial value problem where a wave is driven at the surface $\phi=$ $\phi_{1}(\psi)$ and propagates along the magnetic field lines. Although the derivative of $A$ with respect to $\psi$ and the derivatives of $\Phi$ with respect to $\psi$ and $h$ change when the wave front propagates, the order of magnitude of these derivatives remain the same. On the other hand, we can see from Eq. (13) that the derivative of $h$ with respect to $\psi$ increases. In particular, when the magnetic field is homogeneous, it follows from Eq. (13) that the first derivative is proportional to $\phi$ and the second to $\phi^{2}$. These estimates remain valid by the order of magnitude even when the magnetic field is inhomogeneous. As a result, at some distance from the surface where the wave is driven, the derivatives of $h$ with respect to $\psi$ become so large that the right-hand side of Eq. (11) cannot be neglected even in the case of small viscosity. At this stage of the wave evolution the last two terms in the square brackets on the right-hand side of Eq. (11) strongly dominate the other two terms, which can be neglected. Hence, Eq. (11) reduces to the approximate form

$$
\begin{aligned}
& \frac{\partial^{2} \Phi}{\partial t^{2}}- V_{0}^{2} \frac{\partial^{2} \Phi}{\partial h^{2}}-\frac{\sigma \Phi}{\mu_{0} B_{0}^{2}}= \\
& \frac{v B^{2}}{B_{0}^{2}} \frac{\partial}{\partial t}\left[\frac{\partial^{2} h}{\partial \psi^{2}} \frac{\partial \Phi}{\partial h}+\left(\frac{\partial h}{\partial \psi}\right)^{2} \frac{\partial^{2} \Phi}{\partial h^{2}}\right] .
\end{aligned}
$$

When $v=0$ this equation is the Klein-Gordon equation with constant coefficients. It admits a solution $\Phi=\Phi_{0} \exp (\mathrm{i} k h-\mathrm{i} \omega t)$, where $\Phi_{0}$ is a constant, and $k$ and $\omega$ are related by

$\omega^{2}=V_{0}^{2} k^{2}-\frac{\sigma}{\mu_{0} B_{0}^{2}}$.

We see that waves with arbitrary frequency can propagate when $\sigma>0$, while only waves with $\omega>\omega_{\text {cut }}$ can propagate when $\sigma<0$, where the cutoff frequency $\omega_{\text {cut }}$ is defined by

$\omega_{\mathrm{cut}}^{2}=-\frac{\sigma}{\mu_{0} B_{0}^{2}}$.

Finally, when $\sigma=0$ we obtain $\omega^{2}=V_{0}^{2} k^{2}$ and, again, waves with arbitrary frequency can propagate.

Now we proceed to studying Eq. (15). We start from the simplest case where $\sigma=0$. In that case we immediately obtain from Eq. (15) that

$A=A_{0}(\psi)+A_{1}(\psi) \int_{0}^{\phi} \frac{B_{0}^{2}}{B^{2}} \mathrm{~d} \phi^{\prime}$,

where $A_{0}(\psi)$ and $A_{1}(\psi)$ are arbitrary functions. In particular, taking $A_{1}(\psi)=0$ we obtain that $A$ and consequently $\rho$ is independent of $\phi$.

Then we assume that $\sigma \neq 0$. We search for the solution to this equation in the form

$B^{2} \frac{\partial A}{\partial \phi}=S(A, \psi)$
Substituting this expression in Eq. (15) and using Eq. (13) we obtain

$A^{3} S \frac{\partial S}{\partial A}=\sigma(\psi)$

It follows from this equation that

$B^{2} \frac{\partial A}{\partial \phi}= \pm \sqrt{C(\psi)-\frac{\sigma(\psi)}{A^{2}}}$

where $C(\psi)$ is an arbitrary function. The general solution to this equation is

$$
\sqrt{A^{2} C(\psi)-\sigma(\psi)}= \pm C(\psi) \int_{\phi_{0}(\psi)}^{\phi} \frac{\mathrm{d} \phi^{\prime}}{B^{2}\left(\phi^{\prime}, \psi\right)}
$$

when $C(\psi) \neq 0$, where $\phi_{0}(\psi)$ is an arbitrary function. When $C(\psi)=0$, the general solution to Eq. (22) is

$A^{2}= \pm 2 \sqrt{-\sigma(\psi)} \int_{\bar{\phi}_{0}(\psi)}^{\phi} \frac{\mathrm{d} \phi^{\prime}}{B^{2}\left(\phi^{\prime}, \psi\right)}$,

where $\bar{\phi}_{0}(\psi)$ is an arbitrary function. Obviously, this expression is only valid when $\sigma(\psi)<0$.

\section{Alfvén wave damping due to phase mixing}

In this section we study the damping of Alfvén waves due to phase mixing. To derive the expression describing the wave decay we slightly modified the method developed by Heyvaerts \& Priest (1983) and Ruderman (1999) to study the phase mixing of standing Alfvén waves. We impose the boundary condition

$u=u_{0}(t, \psi)$ at $\phi=\phi_{1}(\psi)$.

This boundary condition is valid for any $t$ and $\psi$. Now we assume that $u$ and consequently $\Phi$ is proportional to $\exp (-\mathrm{i} \omega t)$. Then Eq. (16) transforms to

$V_{0}^{2} \frac{\partial^{2} \Phi}{\partial h^{2}}+\lambda^{2} \Phi=\frac{i \omega v B^{2}}{B_{0}^{2}}\left[\frac{\partial^{2} h}{\partial \psi^{2}} \frac{\partial \Phi}{\partial h}+\left(\frac{\partial h}{\partial \psi}\right)^{2} \frac{\partial^{2} \Phi}{\partial h^{2}}\right]$,

where

$\lambda^{2}=\omega^{2}+\frac{\sigma}{\mu_{0} B_{0}^{2}}$

Below we assume that $\lambda^{2}>0$. Using Eqs. (10) and (13) we transform Eq. (25) to

$\Phi=A^{-1}(0, \psi) u_{0}(\psi) \equiv \Phi_{0}(\psi) \quad$ at $h=0$.

The variable $h$ is a spatial variable related to the distance from the surface $\phi=\phi_{1}(\psi)$ to a point in a particular magnetic field line. Below we assume that the characteristic distance of damping due to phase mixing is much greater than the characteristic distance of variation of $\Phi$ with respect to $h$. This assumption is not very restrictive. Actually, the dependence of $\Phi$ on $h$ is approximately harmonic with the wave number $k$. Then the characteristic distance of variation of $\Phi$ with respect to $h$ is $1 / k$, which is approximately $1 / 6$ of the wavelength. Hence, our assumption is valid even when the damping length is of the order of the wavelength. Now, in accordance with our assumption, we introduce a "slow" variable $h_{1}=\epsilon h$, where $\epsilon \ll 1$. 
To characterise the viscosity magnitude we introduce the Reynolds number $\operatorname{Re}=l V_{0} / v$, where $l$ is the characteristic spatial scale of variation of equilibrium quantities. We assume that the characteristic wavelength is also $l$. Below we also assume that the viscosity is weak, $\operatorname{Re} \gg 1$. Now we introduce the scaled kinematic viscosity $\bar{v}=\operatorname{Re} v$. The relation between $\epsilon$ and $\operatorname{Re}$ will be defined later. Then Eq. (26) is rewritten as

$\epsilon^{2} \frac{\partial^{2} \Phi}{\partial h_{1}^{2}}+\frac{\lambda^{2}}{V_{0}^{2}} \Phi=\frac{\mathrm{i} \omega \bar{v} B^{2}}{V_{0}^{2} B_{0}^{2} \operatorname{Re}}\left[\frac{\partial^{2} h_{1}}{\partial \psi^{2}} \frac{\partial \Phi}{\partial h_{1}}+\left(\frac{\partial h_{1}}{\partial \psi}\right)^{2} \frac{\partial^{2} \Phi}{\partial h_{1}^{2}}\right]$.

Equation (13) defines $\phi$ as an implicit function of $h$ and, consequently, $h_{1}$. Hence, $\partial \Phi / \partial h_{1}$ and $\partial^{2} \Phi / \partial h_{1}^{2}$ must be considered as functions of $h_{1}$ and $\psi$. Now we use the standard WKB method and search for the solution to this equation in the form

$\Phi=Q\left(h_{1}, \psi\right) \exp \left[\mathrm{i} \epsilon^{-1} \Theta\left(h_{1}, \psi\right)\right]$

(see e.g. Bender \& Orszag 1999). Substituting this expression in Eq. (29) we obtain

$$
\begin{gathered}
\epsilon^{2} \frac{\partial^{2} Q}{\partial h_{1}^{2}}+2 \mathrm{i} \epsilon \frac{\partial Q}{\partial h_{1}} \frac{\partial \Theta}{\partial h_{1}}+\mathrm{i} \epsilon Q \frac{\partial^{2} \Theta}{\partial h_{1}^{2}}-Q\left(\frac{\partial \Theta}{\partial h_{1}}\right)^{2} \\
+\frac{\lambda^{2}}{V_{0}^{2}} Q=\frac{\mathrm{i} \omega \bar{v} B^{2}}{V_{0}^{2} B_{0}^{2} \operatorname{Re}}\left\{\frac{\partial^{2} h_{1}}{\partial \psi^{2}}\left(\frac{\partial Q}{\partial h_{1}}+\mathrm{i} \epsilon^{-1} Q \frac{\partial \Theta}{\partial h_{1}}\right)\right. \\
+\left(\frac{\partial h_{1}}{\partial \psi}\right)^{2}\left[\frac{\partial^{2} Q}{\partial h_{1}^{2}}+2 \mathrm{i} \epsilon^{-1} \frac{\partial Q}{\partial h_{1}} \frac{\partial \Theta}{\partial h_{1}}\right. \\
\left.\left.+\mathrm{i} \epsilon^{-1} Q \frac{\partial^{2} \Theta}{\partial h_{1}^{2}}-\epsilon^{-2} Q\left(\frac{\partial \Theta}{\partial h_{1}}\right)^{2}\right]\right\} .
\end{gathered}
$$

First we assume that the right-hand side is much smaller than the two largest terms on the left-hand side of this equation, which are the last and next-to-last terms. Then we collect the terms of the order of unity in Eq. (31) to obtain

$\left(\frac{\partial \Theta}{\partial h_{1}}\right)^{2}=\frac{\lambda^{2}}{V_{0}^{2}}$.

This approximation is usually called the approximation of geometrical optics (e.g. Bender \& Orszag 1999). It determines the shape of rays along which the waves propagate. Only considering waves propagating in the positive $\phi$-direction, from Eq. (32) we obtain

$\Theta=\frac{h_{1} \lambda(\psi)}{V_{0}}$,

where we arbitrarily take $\Theta=0$ at $h_{1}=0$. In the next order approximation we collect terms of the order $\epsilon$. This approximation is usually called the approximation of physical optics. It determines the spatial evolution of the wave amplitude. The righthand side of Eq. (31) describes the viscous wave damping due to phase mixing. Hence, we define the relation between Re and $\epsilon$ in such a way that the right-hand side of Eq. (31) contributes in the approximation of physical optics. In accordance with this we take $\operatorname{Re}=\epsilon^{-3}$. Then we obtain

$2 \frac{\partial Q}{\partial h_{1}} \frac{\partial \Theta}{\partial h_{1}}+Q \frac{\partial^{2} \Theta}{\partial h_{1}^{2}}=-\frac{\omega \bar{v} B^{2} Q}{V_{0}^{2} B_{0}^{2} \operatorname{Re}}\left(\frac{\partial h_{1}}{\partial \psi}\right)^{2}\left(\frac{\partial \Theta}{\partial h_{1}}\right)^{2}$.

Using Eq. (33) and returning to the original variables we transform Eq. (34) to

$\frac{\partial Q}{\partial h}=-\Upsilon(h, \psi) Q, \quad \Upsilon(h, \psi)=\frac{\omega \nu \lambda B^{2}}{2 B_{0}^{2} V_{0}^{3}}\left(\frac{\partial h}{\partial \psi}\right)^{2}$.
Obviously we can assume that $Q$ is real. Equation (35) determines the spatial evolution of the Alfvén wave amplitude. It will be studied below in various equilibria. We will also use the expressions for the velocity and magnetic field perturbation. Using Eqs. (10) and (30) we obtain in the leading order approximation with respect to $\epsilon$

$u=\rho^{-1 / 4} Q \exp \left[\mathrm{i}\left(h \lambda / V_{0}-\omega t\right)\right]$.

Then, using Eqs. (3), (6), and (13) yields

$b=-\mu_{0}^{1 / 2} \rho^{1 / 4} \omega^{-1} \lambda Q \exp \left[\mathrm{i}\left(h \lambda / V_{0}-\omega t\right)\right]$.

\section{Alfvén wave phase mixing in equilibria with straight magnetic field lines}

In this section we assume that the magnetic field is in the $z$ direction. It follows from Eq. (1) that $B_{z}=$ const. We take $B_{z}=B_{0}$. Then $\phi=z$ and $\psi=x$. Hence, in this section we use $x$ and $z$ instead of $\psi$ and $\phi$. We also assume that $v$ is independent of $z$, and take the lower limit in the expression Eq. (13) equal to zero $\left(\phi_{1}(\psi)=0\right)$.

\subsection{Alfvén speed not varying along the magnetic field direction}

We assume that $\rho=A^{-4}$ is independent of $z$. It then follows from Eq. (15) that $\sigma(x)=0$. To satisfy Eq. (23) we also take $C(x)=0$. Now it follows that $V_{\mathrm{A}}$ is independent of $z$, i.e. it does not vary along the magnetic field direction. Then it follows from Eq. (13) that $h=z V_{0} / V_{\mathrm{A}}(x)$. Using this result we obtain

$\Upsilon(h, x)=\frac{\omega^{2} v h^{2}}{2 V_{\mathrm{A}}^{2} V_{0}^{3}}\left(\frac{\mathrm{d} V_{\mathrm{A}}}{\mathrm{d} x}\right)^{2}$,

where we have taken into account that $\lambda=\omega$ when $\sigma=0$. Substituting this result in Eq. (35) and integrating the obtained equation yields

$Q=Q_{0}(x) \exp \left[-\frac{\omega^{2} v h^{3}}{6 V_{\mathrm{A}}^{2} V_{0}^{3}}\left(\frac{\mathrm{d} V_{\mathrm{A}}}{\mathrm{d} x}\right)^{2}\right]$,

where $Q_{0}(x)$ is the value of $Q$ at $h=0$. Using Eqs. (10), (25), (30), and (33), we eventually arrive at

$u=u_{0}(x) \exp \left[-\frac{\omega^{2} v z^{3}}{6 V_{\mathrm{A}}^{5}}\left(\frac{\mathrm{d} V_{\mathrm{A}}}{\mathrm{d} x}\right)^{2}\right] \cos (\omega t)$.

We see that the logarithm of the wave amplitude is proportional to $-z^{3}$, and the damping length is proportional to $\mathrm{Re}^{1 / 3}$. These are familiar results (e.g. Heyvaerts \& Priest 1983).

\subsection{Linear variation of Alfvén speed in the magnetic field direction}

In this section we consider the case where $C(\psi)=0$. Since in this case $\sigma(x)<0$, to have $\lambda^{2}>0$ we assume that $\omega^{2}>$ $\max |\sigma(x)| / \mu_{0} B_{0}^{2}$. It follows from the relation $A=\rho^{-1 / 4}$ and Eq. (24) that

$\rho=\frac{\rho_{0}(x)}{[1+z \kappa(x)]^{2}}, \quad V_{\mathrm{A} 0}^{2}(x)=\frac{B_{0}^{2}}{\mu_{0} \rho_{0}(x)}$,
$V_{\mathrm{A}}(x, z)=V_{\mathrm{A} 0}(x)[1+z \kappa(x)]$ 
where $\rho_{0}(x)$ is an arbitrary positive function and $\kappa(x)=$ $-1 / \bar{\phi}_{0}(x)$. Below we assume that $\kappa=$ const and $\kappa>0$. Substituting this expression for $V_{\mathrm{A}}$ in Eq. (13) yields

$h=\frac{V_{0} \ln (1+z \kappa)}{\kappa V_{\mathrm{A} 0}(x)}$.

Using this result we obtain

$\Upsilon(h, x)=\frac{\omega v \lambda h^{2}}{2 V_{0}^{3} V_{\mathrm{A} 0}^{2}}\left(\frac{\mathrm{d} V_{\mathrm{A} 0}}{\mathrm{~d} x}\right)^{2}$.

Substituting this result in the first equation in Eq. (35) and integrating the obtained equation yields

$Q=Q_{0}(x) \exp \left\{-\alpha[\ln (1+\kappa z)]^{3}\right\}$,

where

$\alpha=\frac{\omega \nu \lambda}{6 \kappa^{3} V_{\mathrm{A} 0}^{5}}\left(\frac{\mathrm{d} V_{\mathrm{A} 0}}{\mathrm{~d} x}\right)^{2}$.

Using Eqs. (10), (25), (30), and (33), we arrive at

$u=u_{0}(x) \exp \left\{-\alpha[\ln (1+\kappa z)]^{3}\right\} \cos (\omega t)$

Since $\ln (1+\kappa z) \approx \kappa z$ when $\kappa z \ll 1$, for small $z$ this equation reduces to the form similar to Eq. (40). However when $k z \gtrsim 1$ the density decrease in the magnetic field direction makes the wave damping due to phase mixing less efficient. This result is somewhat similar to that obtained by Ruderman et al. (1998). While the analysis by Ruderman et al. (1998) was carried out under the assumption that the wavelength is much smaller than the characteristic spatial scale of the equilibrium quantity variation, we emphasise that the results of this paper are valid even when these two quantities are of the same order.

\subsection{Alfvén speed is a quadratic function of distance along the magnetic field}

Now we consider the case where $C(x) \neq 0$. Below we assume that $\phi_{0}(x)=0$ and $\sigma(x)>0$. We also assume that $C^{2}(x) / \sigma(x)=$ const. It follows from Eq. (23) that

$\rho^{-1 / 2}=A^{2}=\frac{z^{2} C(x)}{B_{0}^{4}}+\frac{\sigma(x)}{C(x)}$.

Using this result we obtain

$V_{\mathrm{A}}=V_{\mathrm{A} 0}(x)\left(1+k^{2} z^{2}\right)$

where

$k^{2}=\frac{C^{2}(x)}{B_{0}^{4} \sigma(x)}=$ const.,$\quad V_{\mathrm{A} 0}(x)=\frac{B_{0} \sigma(x)}{C(x) \sqrt{\mu_{0}}}$.

It follows from Eq. (13) that

$h=\frac{V_{0} \arctan (k z)}{k V_{\mathrm{A} 0}(x)}$.

This equation can be rewritten as

$Q=Q_{0}(x) \exp \left\{-\beta[\arctan (k z)]^{3}\right\}$,

A122, page 6 of 11 where

$\beta=\frac{\omega v \lambda}{6 k^{3} V_{\mathrm{A} 0}^{5}}\left(\frac{\mathrm{d} V_{\mathrm{A} 0}}{\mathrm{~d} x}\right)^{2}$.

Using Eqs. (10), (25), (30), and (33), we arrive at

$u=u_{0}(x) \exp \left\{-\beta[\arctan (k z)]^{3}\right\} \cos (\omega t)$.

Since $\arctan (k z) \approx k z$ when $k z \ll 1$, for small $z$ this equation reduces to the form similar to Eq. (40). When $k z \gtrsim 1$ the density decrease in the magnetic field direction reduces the wave damping rate due to phase mixing. It is also worth noting that now the wave amplitude does not tend to zero as $z \rightarrow \infty$. Instead it tends to $u_{0}(x) \exp \left(-\beta \pi^{3} / 8\right)$. Again a similar result was obtained by Ruderman et al. (1998) in their study of damping due to phase mixing of Alfvén waves with short wavelength. Finally, we notice that the results obtained in this subsection are only valid on a restricted time interval. At fixed $x$, a perturbation launched at $z=0$ and $t=0$ arrives at infinity at finite time equal to $\pi\left[2 k V_{\mathrm{A} 0}(x)\right]^{-1}$. This is an unphysical behaviour implying that we only can consider the wave evolution on a finite interval of $z$.

\section{Alfvén wave phase mixing in equilibria with divergent magnetic field lines}

In this section we study the Alfvén wave phase mixing in equilibria with the exponentially divergent magnetic field lines similar to those considered by Ruderman et al. (1998) and Smith et al. (2007).

\subsection{Equilibrium configuration}

We take

$\psi=H \mathrm{e}^{-z / H} \sin (x / H), \quad \phi=-H \mathrm{e}^{-z / H} \cos (x / H)$,

where $H$ is a constant. Then we obtain

$B_{x}=B_{0} \mathrm{e}^{-z / H} \sin (x / H), \quad B_{z}=B_{0} \mathrm{e}^{-z / H} \cos (x / H)$.

It follows from Eqs. (54) and (55) that

$B^{2}=\frac{B_{0}^{2}}{H^{2}}\left(\phi^{2}+\psi^{2}\right)$.

It is straightforward to see that the distance between two neighbouring magnetic field lines exponentially increases with height. The sketch of the equilibrium is shown in Fig. 1.

We consider the wave propagation in a region that is narrow at its base. It is bounded by the magnetic field lines determined by $\psi= \pm \psi_{b}, \psi_{b} \ll H$. The equations for these magnetic field lines are

$x= \pm H \arcsin \frac{\psi_{b} \mathrm{e}^{z / H}}{H}, \quad z \in\left[0, H \ln \left(H / \psi_{b}\right)\right]$.

The boundary magnetic field lines reach their maximum height $z=H \ln \left(H / \psi_{b}\right)$ corresponding to $\phi=0$ at $x= \pm \pi H / 2$, and then go down. Below we take $\phi_{1}=-\sqrt{H^{2}-\psi_{b}^{2}}$ and restrict the region where we consider the wave propagation by $\phi_{1} \leq \phi \leq \phi_{2}<0$. Then the maximum width of this region is

$L_{m}=-2 H \arctan \frac{\psi_{b}}{\phi_{2}}$.

, 


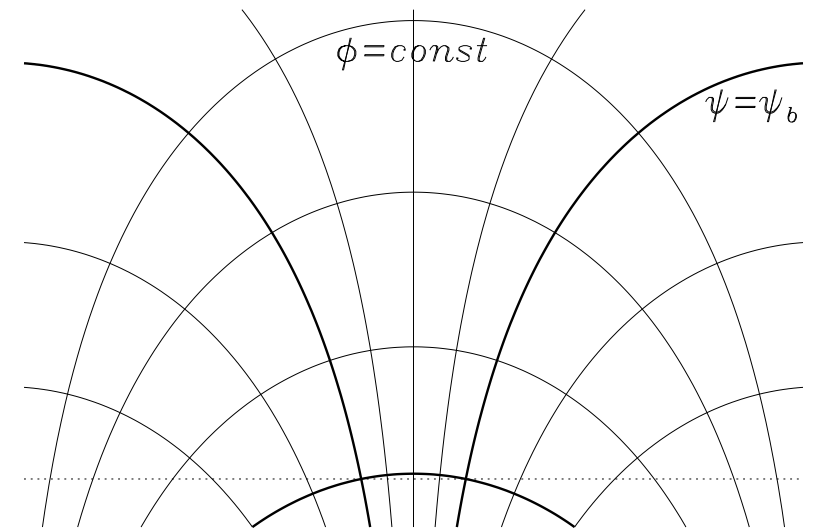

Fig. 1. Equilibrium with divergent magnetic field lines. The vertical curves are the magnetic field lines defined by the equation $\psi=$ const. The horizontal curves are defined by the equation $\phi=$ const. The thick vertical curves are the boundaries of the region where the wave propagation is considered. They are defined by the equation $\psi= \pm \psi_{b}$. The thick horizontal curve is defined by the equation $\phi=\phi_{1}$. It is assumed that the wave is driven at this line. The dotted line shows the level $z=0$.

We note that the lower boundary of the region determined by the equation $\phi=\phi_{1}$ crosses the boundary magnetic field lines at $z=0$. It is assumed that the perturbations are driven at this lower boundary.

Now we assume that $\sigma=0$ and $\rho$ is independent of $\phi$, meaning that the plasma density does not vary along the magnetic field lines. Hence, the Alfvén velocity variation along the magnetic field lines is only due to the magnetic field magnitude variation.

\subsection{Alfvén wave phase mixing}

\subsubsection{Qualitative analysis}

We first point out one remarkable property of Eq. (7) with the zero right-hand side, which corresponds to $v=0$. When the plasma density $\rho$ is independent of $\phi$ the variable substitution Eq. (13) reduces this equation to the wave equation with constant coefficient, which is Eq. (16) with $v=0$ and $\sigma=0$. This is correct for any two-dimensional equilibrium magnetic field, even for a non-potential field. In the latter case $\phi$ is simply the curvilinear coordinate constituting together with the magnetic flux function an orthogonal curvilinear coordinate system.

Recalling that $\rho$ is independent of $\phi$ and $\phi_{1}=-\sqrt{H^{2}-\psi_{b}^{2}}$ in the expression for $h$, Eq. (13), we obtain for the particular equilibrium described in the previous subsection

$h=\frac{H^{2} V_{0}}{\psi V_{\mathrm{A} 0}}\left(\arctan \frac{\phi}{\psi}-\arctan \frac{\phi_{1}}{\psi}\right)$,

where $V_{\mathrm{A} 0}^{2}=B_{0}^{2} / \mu_{0} \rho$. Differentiating this expression yields

$$
\begin{aligned}
\frac{\partial h}{\partial \psi}= & \frac{\psi V_{0} H^{2}}{2 \rho V_{\mathrm{A} 0}} \frac{\mathrm{d}}{\mathrm{d} \psi}\left(\frac{\rho}{\psi^{2}}\right)\left(\arctan \frac{\phi}{\psi}-\arctan \frac{\phi_{1}}{\psi}\right) \\
& -\frac{H^{2} V_{0}}{\psi V_{\mathrm{A} 0}}\left(\frac{\phi}{\phi^{2}+\psi^{2}}-\frac{\phi_{1}}{\phi_{1}^{2}+\psi^{2}}\right) .
\end{aligned}
$$

Below we assume that $\rho$ is an even function of $\psi$. Then it is not difficult to show that $\partial h / \partial \psi \rightarrow 0$ as $\psi \rightarrow 0$. It is convenient to transform Eq. (35) to

$\frac{\partial Q}{\partial \phi}=-\widetilde{\Upsilon}(\phi, \psi) Q, \quad \widetilde{\Upsilon}(\phi, \psi)=\frac{v \omega^{2} B}{2 B_{0} V_{0}^{2} V_{\mathrm{A}}}\left(\frac{\partial h}{\partial \psi}\right)^{2}$.
The solution to this equation is

$Q(\phi, \psi)=Q_{1}(\psi) \exp [-\Lambda(\phi, \psi)]$,

$\Lambda(\phi, \psi)=\int_{\phi_{1}}^{\phi} \widetilde{\Upsilon}\left(\phi^{\prime}, \psi\right) \mathrm{d} \phi^{\prime}$,

where $Q_{1}(\psi)$ is the value of $Q$ at $\phi=\phi_{1}$. We note that we consider $|\psi| \leq \psi_{b} \ll H$. Below we assume that $v$ is independent of $\phi$.

We now consider $z \ll H$. In that case $|x| \ll H$ and $|\phi / \psi| \gg 1$. With these estimates we obtain the approximate expression

$\arctan \frac{\phi}{\psi}-\arctan \frac{\phi_{1}}{\psi} \approx \psi\left(\frac{1}{\phi_{1}}-\frac{1}{\phi}\right)$.

Using this result yields

$\frac{\partial h}{\partial \psi} \approx \frac{V_{0} H^{2}}{2 \rho V_{\mathrm{A} 0}} \frac{d \rho}{\mathrm{d} \psi}\left(\frac{1}{\phi_{1}}-\frac{1}{\phi}\right)$

Then it follows from Eq. (61) that

$\widetilde{\Upsilon}(\phi, \psi) \approx \frac{v \omega^{2} H^{4}}{8 \rho^{2} V_{\mathrm{A} 0}^{3}}\left(\frac{\mathrm{d} \rho}{\mathrm{d} \psi}\right)^{2}\left(\frac{1}{\phi_{1}}-\frac{1}{\phi}\right)^{2}$.

Substituting this expression in Eq. (62) we obtain

$\Lambda(\phi, \psi)=\frac{v \omega^{2} H^{4}}{8 \rho^{2} V_{\mathrm{A} 0}^{3}}\left(\frac{\mathrm{d} \rho}{\mathrm{d} \psi}\right)^{2}\left(\frac{\phi}{\phi_{1}^{2}}-\frac{1}{\phi}-\frac{2}{\phi_{1}} \ln \frac{\phi}{\phi_{1}}\right)$.

Recalling that $|\psi| \ll H$ and $z \ll H$, we can use the approximate expressions

$$
\begin{aligned}
& \phi \approx \phi_{1} \approx-H, \quad \psi \approx x \\
& \frac{1}{\phi_{1}}-\frac{1}{\phi} \approx \frac{1}{H^{2}}\left(z-\frac{\psi_{b}^{2}-\psi^{2}}{2 H}\right) \equiv \frac{\tilde{z}}{H^{2}} .
\end{aligned}
$$

Substituting these expressions in Eq. (66) yields

$\Lambda(\phi, \psi)=\frac{v \omega^{2} \tilde{z}^{3}}{24 \rho^{2} V_{\mathrm{A} 0}^{3}}\left(\frac{\mathrm{d} \rho}{\mathrm{d} x}\right)^{2}$.

Hence, eventually we obtain

$Q(\phi, \psi)=Q_{1}(\psi) \exp \left(-\frac{v \omega^{2} \tilde{z}^{3}}{24 \rho^{2} V_{\mathrm{A} 0}^{3}}\left(\frac{\mathrm{d} \rho}{\mathrm{d} x}\right)^{2}\right)$.

This result qualitatively coincides with that obtained in Sect. 6.1. The only difference is that $z$ is substituted by $\tilde{z}$, which is approximately the distance measured along a magnetic field line from the surface $\phi=\phi_{1}$ where the wave is driven.

Now we consider the wave damping at sufficiently large distances from the source of perturbations. It follows from Eqs. (61) and (62), and that $\partial h / \partial \psi \rightarrow 0$ as $\psi \rightarrow 0$, that there is practically no wave damping when $\psi$ is close to zero. Hence, we assume that $|\psi|$ is not very small, e.g. $|\psi| \geq \frac{1}{2} \psi_{b}$. Then we assume that $\left(\psi_{b} / H\right) \mathrm{e}^{z / H}$ is of the order of unity (we recall that $\left.\psi_{b} \ll H\right)$. We emphasise that this condition is satisfied for very moderate values of $z / H$. For example, if $\psi_{b}=H / 10$, it suffices to assume that $z / H \gtrsim 2$. The condition imposed on $\mathrm{e}^{z / H}$ guaranties that $|x| / H$ is of the order of unity. 
Since $\phi_{1} \approx-H$, while $|\psi| \ll H$, it follows that $\arctan \left(\phi_{1} / \psi\right) \approx-(\pi / 2) \operatorname{sgn}(\psi)$. Then, using Eq. (54), we obtain

$\arctan \frac{\phi}{\psi}-\arctan \frac{\phi_{1}}{\psi} \approx \frac{x}{H}$,

$\frac{\phi}{\phi^{2}+\psi^{2}}-\frac{\phi_{1}}{\phi_{1}^{2}+\psi^{2}} \approx-\frac{\mathrm{e}^{z / H}}{H \cos (z / H)}$.

Using Eqs. (70) and (71) we rewrite Eq. (60) in the approximate form

$\frac{\partial h}{\partial \psi}=-\frac{V_{0} H^{2}}{\psi^{2} V_{\mathrm{A} 0}}\left[\frac{x}{H}\left(1-\frac{\psi}{2 \rho} \frac{\mathrm{d} \rho}{\mathrm{d} \psi}\right)-\tan \frac{x}{H}\right]$.

We recall that $\rho(\psi)$ is assumed to be an even function. In addition we assume that the second derivative of $\rho(\psi)$ is negative, meaning that $\rho(\psi)$ increases for $\psi<0$ and decreases for $\psi>0$. Hence, $\psi(\mathrm{d} \rho / \mathrm{d} \psi)<0$. The signs of $x$ and $\psi$ are the same, meaning that the expression in the square brackets is an odd function of $x$. Consider $x>0$. Then the expression in the square brackets is negative when $|\mathrm{d} \rho / \mathrm{d} \psi|$ is small; however, it is positive if $|\mathrm{d} \rho / \mathrm{d} \psi|$ is sufficiently large. We assume that the latter is true and, moreover, we assume that the expression in the square brackets is of the order of unity when $|x| / H$ is of the order of unity. Then we obtain

$\left|\frac{\partial h}{\partial \psi}\right| \simeq \frac{V_{0} H^{2}}{\psi^{2} V_{\mathrm{A} 0}}=\frac{V_{0} \mathrm{e}^{2 z / H}}{V_{\mathrm{A} 0} \sin ^{2}(x / H)}=\frac{V_{0} H^{2}}{V_{\mathrm{A} 0} \phi^{2}} \cot ^{2} \frac{x}{H}$.

Since $|\cot (x / H)|$ is of the order of unity, it follows that

$$
\begin{aligned}
\Lambda(\phi, \psi) & \simeq \frac{v \omega^{2} H^{4}}{2 V_{\mathrm{A}} V_{\mathrm{A} 0}^{2}} \int_{\phi_{1}}^{\phi} \frac{\mathrm{d} \phi^{\prime}}{\phi^{\prime 4}} \\
& \simeq-\frac{v \omega^{2} H^{4}}{6 V_{\mathrm{A}} V_{\mathrm{A} 0}^{2} \phi^{3}}=\frac{v \omega^{2} H \mathrm{e}^{3 z / H}}{6 V_{\mathrm{A}} V_{\mathrm{A} 0}^{2} \cos ^{3}(x / H)} .
\end{aligned}
$$

This result is similar to the corresponding result obtained by Smith et al. (2007) that the decrement is proportional to $\mathrm{e}^{3 z / H}$.

It is expedient to study the wave damping when the wavelength is much smaller than $H$. It is shown in Appendix A that the expression for the decrement derived in this paper coincides with that obtained by Smith et al. (2007) if we taken the density independent of the height in the latter.

\subsubsection{Numerical results}

To characterise the efficiency of the wave damping we calculate the variation of the wave energy flux with the height. The density of the wave energy flux is $\frac{1}{2} V_{\mathrm{A}}\left(\rho v^{2}+b^{2} / \mu_{0}\right)$, and it is in the equilibrium magnetic field direction. Using Eq. (54) we obtain that the square of the elementary length along the surface $\phi=$ const. is

$\mathrm{d} x^{2}+\mathrm{d} z^{2}=\frac{H^{2} \mathrm{~d} \psi^{2}}{\phi^{2}+\psi^{2}}$.

Equations (36) and (37) give the complex-valued expressions for $u$ and $b$. To obtain the real expressions we need to take the real parts of these expressions. In addition, we take the average value of the wave energy density flux over the wave period. Then, using Eq. (54), we obtain that the average wave energy flux through the surface $\phi=$ const. per unit length in the $y$-direction between $\psi=-\psi_{b}$ and $\psi=\psi_{b}$ is

$\Pi=\frac{B_{0}}{2 \sqrt{\mu_{0}}} \int_{-\psi_{b}}^{\psi_{b}} Q^{2} \mathrm{~d} \psi$
For the numerical study we adopted the following model. We took

$\rho=\rho_{0}\left\{1+\varsigma \exp \left[-3\left(\psi / \psi_{b}\right)^{2}\right]\right\}$,

meaning that the density enhancement is almost completely confined in the magnetic tube bounded by the magnetic field lines $\phi= \pm \psi_{b}$. We also took

$Q_{1}(\psi)=\hat{Q} \begin{cases}1-\left(\psi / \psi_{b}\right)^{2}, & |\psi| \leq \psi_{b}, \\ 0, & |\psi|>\psi_{b},\end{cases}$

where $\rho_{0}$ and $\hat{Q}$ are constants. We put $V_{0}=B_{0} / \sqrt{\mu_{0} \rho_{0}}$ and introduce the dimensionless frequency $\varpi=\omega H /\left(2 \pi V_{0}\right)$, meaning that the wave period is $H /\left(\varpi V_{0}\right)$. The surface $\phi=\hat{\phi}=$ const. intersects the vertical axis at $z=z_{\phi} \equiv-H \ln (-\hat{\phi} / H)$. The surface $\phi=\phi_{1}$ at which the wave is driven intersects the vertical axis at $z=z_{1} \equiv-H \ln \left(-\phi_{1} / H\right)$. We calculated the dependence of the relative energy flux, $\Delta=\Pi / \Pi_{0}$, where $\Pi_{0}$ is the energy flux through the surface $\phi=\phi_{1}$, on $s=\left(z_{\phi}-z_{1}\right) / H$. Using Eqs. (60)(62), (76), (77), and assuming that $v$ is constant, we obtain

$$
\begin{aligned}
\Delta= & \frac{\Pi}{\Pi_{0}}=\frac{15}{8} \int_{0}^{1}\left(1-\xi^{2}\right)^{2} \mathrm{e}^{-2 \Lambda} \mathrm{d} \xi \\
\Lambda= & 2 \pi^{2} \chi^{-1} \xi_{b}^{-4}\left(1+\varsigma \mathrm{e}^{-3 \xi^{2}}\right)^{3 / 2} \int_{\eta_{1}}^{\eta}\left[\left(\frac{1}{\xi^{2}}\right.\right. \\
& \left.+\frac{3 \varsigma}{\mathrm{e}^{3 \xi^{2}}+\varsigma}\right)\left(\arctan \frac{\eta^{\prime}}{\xi \xi_{b}}-\arctan \frac{\eta_{1}}{\xi \xi_{b}}\right) \\
& \left.+\frac{\xi_{b}}{\xi}\left(\frac{\eta^{\prime}}{\eta^{\prime 2}+\xi^{2} \xi_{b}^{2}}-\frac{\eta_{1}}{\eta_{1}^{2}+\xi^{2} \xi_{b}^{2}}\right)\right]^{2} \mathrm{~d} \eta^{\prime},
\end{aligned}
$$

where $\xi=\psi / \psi_{b}, \xi_{b}=\psi_{b} / H, \eta=\phi / H, \eta_{1}=\phi_{1} / H=-\sqrt{1-\xi_{b}^{2}}$, $\chi=\operatorname{Re} / \varpi^{2}$, and $\operatorname{Re}=H V_{0} / v$ is the Reynolds number. It follows from Eqs. (78) and (79) that $\Delta(s)$ depends on three dimensionless parameters: $\chi, \xi_{b}$, and $\varsigma$. In our calculations we took $\varsigma=4$, meaning that the ratio of the maximum density at $x=0$ to the density far from the region where the wave propagates is 5 . We calculated the dependence of $\Delta$ on $s$ for fixed $\chi$ and various values of $\xi_{b}$. The results of these calculations are shown in Fig. 2 for $\chi=2 \times 10^{7}$. We can see in Fig. 2 that the wave damping becomes more efficient when $\xi_{b}$ decreases. We obtained similar results for other values of $\chi$.

We now apply the numerical results to the damping of Alfvén waves in coronal plumes. Using the WKB method Smith et al. (2007) studied the Alfvén wave damping owing phase mixing in a two-dimensional equilibrium with exponentially divergent magnetic field lines and density exponentially decreasing with the height. It follows from their analysis that the effect of the density variation with the height can be neglected if the density scale height $H_{\rho}$ is at least twice as high as the magnetic field scale height $H, H_{\rho} \geq 2 H$. If we take as a typical value $H_{\rho}=60 \mathrm{Mm}$, then it follows that our model is applicable if $H \leq 30 \mathrm{Mm}$. We take the maximum possible value of the magnetic field scale height $H=30 \mathrm{Mm}$. The typical plume size at the base is $5 \mathrm{Mm}$ (e.g. DeForest et al. 1997). Since $\psi \approx x$ at $z=0$, this corresponds to $\xi_{b}=\psi_{b} / H=3 / 30=1 / 10$. We take the electron number density at the centre of the plume equal to $10^{15} \mathrm{~m}^{-3}$. Then, for $s=4$, the electron number density far from the plume is $2 \times 10^{14} \mathrm{~m}^{-3}$. Finally, we take $B_{0}=10 \mathrm{G}$. Then we obtain for the Alfvén speed at the centre of the plume $V_{0} \approx 700 \mathrm{~km} \mathrm{~s}^{-1}$.

If we take the plasma temperature equal to $10^{6} \mathrm{~K}$, then the classical theory gives $\tau_{i} \approx 1 \mathrm{~s}$ and $v_{0} \approx 10^{10} \mathrm{~m}^{2} \mathrm{~s}^{-1}$ for the ion 


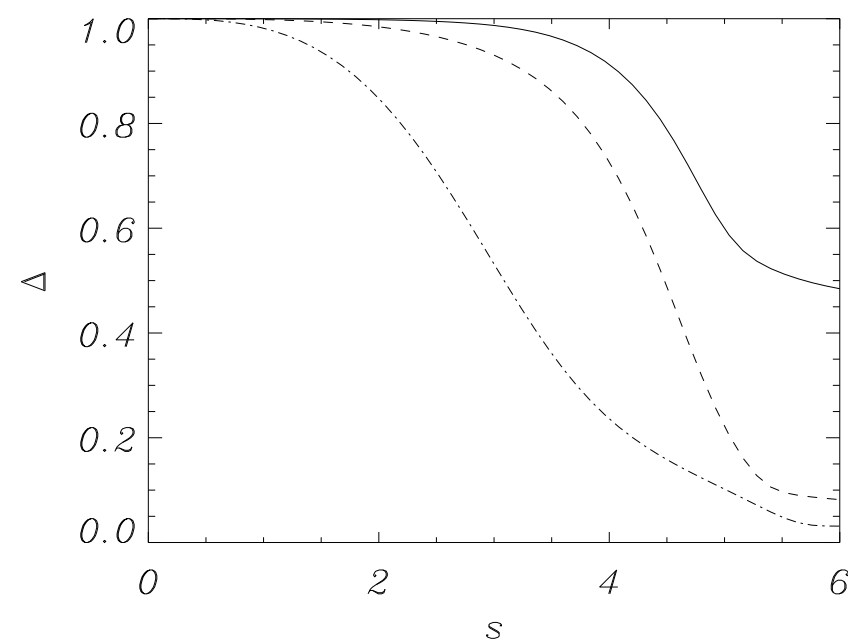

Fig. 2. Dependence of $\Delta$ on $s$ for $\chi=2 \times 10^{7}$ and various values of $\xi_{b}$. The solid, dashed and dash-dotted lines correspond to $\xi_{b}=1 / 10$, $\xi_{b}=1 / 30$, and $\xi_{b}=1 / 100$, respectively.

collision time and for the first coefficient of viscosity in the full Braginskii's viscosity tensor (Spitzer 1962; Braginskii 1965). However, the viscosity tensor in the solar corona is strongly anisotropic due to the presence of magnetic field. The first term in the expression for the Braginskii's viscosity tensor describes the volume viscosity that does not affect Alfvén waves. The Alfvén wave damping is related to the shear viscosity. The shear viscosity is smaller than $v_{0}$ by factor $\left(\tau_{i} \omega_{i}\right)^{-2}$, where $\omega_{i}$ is the ion gyrofrequency. For $B_{0}=10 \mathrm{G}$ we obtain $\omega_{i} \approx 10^{5} \mathrm{~s}^{-1}$. As a result we obtain $v \approx\left(\tau_{i} \omega_{i}\right)^{-2} v_{0} \approx 1 \mathrm{~m}^{2} \mathrm{~s}^{-1}$. But this estimate must be taken with caution. It is believed that the turbulence can strongly enhance shear viscosity. Therefore it seems reasonable to consider $v$ as a free parameter.

As we can see from the expression for $\chi$, the efficiency of the Alfvén wave damping strongly depends on the wave period. The shorter the wave period the stronger the damping is. The initial wavelength is given by $2 \pi V_{0} / \omega=H / \varpi$. If we take $v=1 \mathrm{~m}^{2} \mathrm{~s}^{-1}$ given by the classical theory, then we obtain $\operatorname{Re}=H V_{0} / v=$ $2 \times 10^{13}$. A simple estimate shows that for this value of $\mathrm{Re}$, the plume size equal to $6 \mathrm{Mm}$, and the wave period equal to $30 \mathrm{~s}$, the Afvén wave damping within a few scale heights if practically negligible.

In Fig. 3 the dependence of the relative wave energy flux $\Delta$ on the dimensionless height $s$ is shown for $\xi_{b}=1 / 10$ and four values of the parameter $\chi$. We can see that in order for at least half of the wave energy to dissipate before the wave reaches the height $6 H$, a value $\chi \leq 2 \times 10^{7}$ is needed. For waves with the period equal to $60 \mathrm{~s}$, which corresponds to $\varpi=1$, this implies $\operatorname{Re} \leq 2 \times 10^{7}$; i.e., the shear viscosity $v$ must be reduced by 6 orders of magnitude in comparison with the value given by the classical plasma theory. We can relax this estimate if we decrease the wave period. To get $\chi=2 \times 10^{7}$ when $\mathrm{Re}=2 \times 10^{13}$ we need to take the period equal to $0.06 \mathrm{~s}$. Obviously, the waves with such a small period are not described by our theory. They are not even described by the MHD because their period is less than the ion collision time.

Another way to increase the efficiancy of wave damping at a fixed Reynolds number is to reduce $\xi_{b}$. We calculated the dependence of $\chi$ on $\xi_{b}$ defined by the condition that the wave energy flux reduces twice at $z=6 H$. This dependence is shown in Fig. 4. We see that in order to have the half wave energy damped before the wave reaches the height $z=6 H$ when $\chi=2 \times 10^{13}$, which

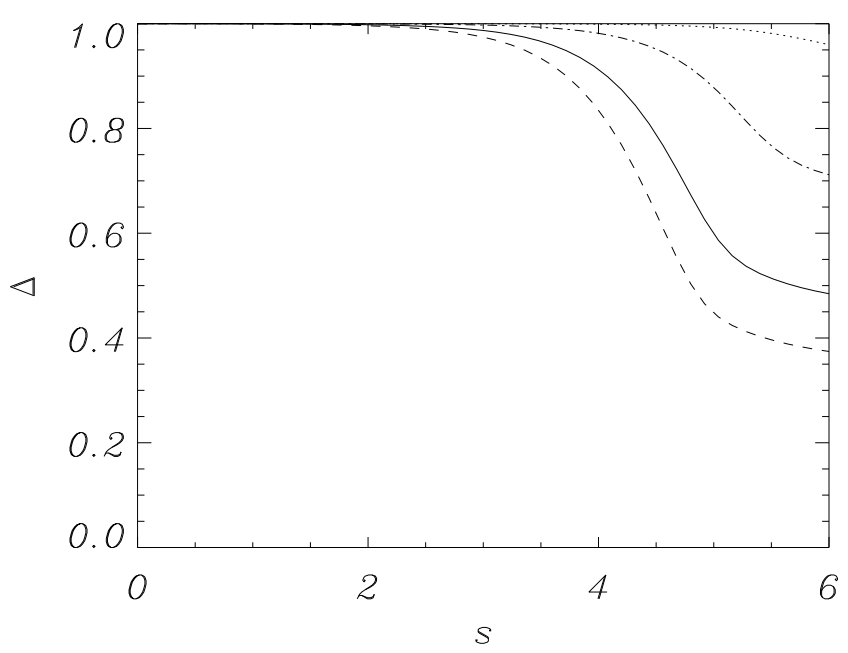

Fig. 3. Dependence of $\Delta$ on $s$ for $\xi_{b}=1 / 10$ and various values of $\chi$. The dashed, solid, dash-dotted, and dotted lines correspond to $\chi=10^{7}$, $\chi=2 \times 10^{7}, \chi=10^{8}$, and $\chi=2 \times 10^{9}$, respectively.

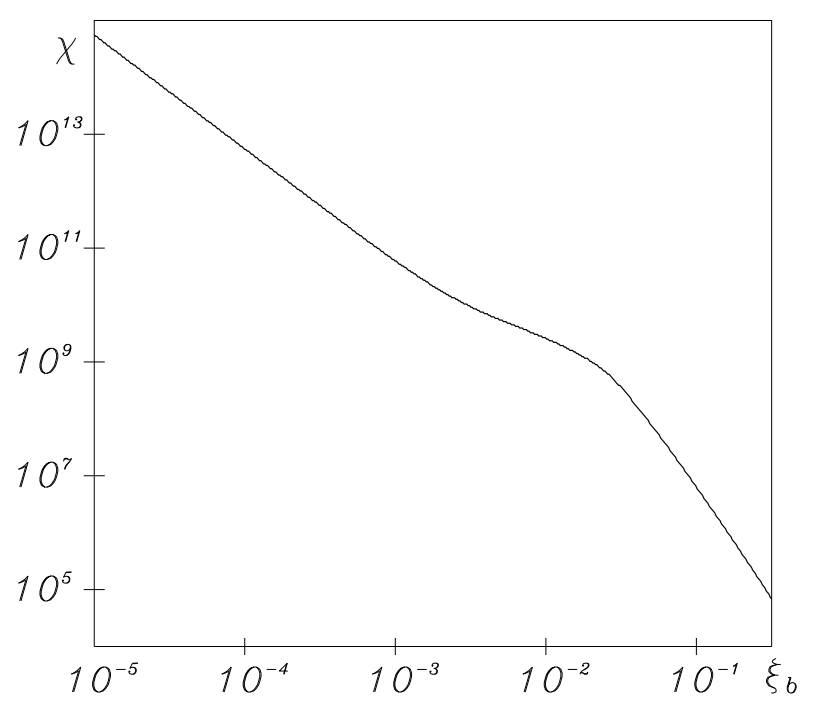

Fig. 4. Dependence of $\chi$ on $\xi_{b}$ defined by the condition that the wave energy flux reduces twice at the height $z=6 H$, i.e. $\Delta(6)=\frac{1}{2}$. We note the logarithmic scale of the axes.

corresponds to $\mathrm{Re}=2 \times 10^{13}$ for the wave period $60 \mathrm{~s}$, we need to take $\xi_{b} \simeq 10^{-4}$. This value of $\xi_{b}$ corresponds to the plume width at its base of the order of $5 \mathrm{~km}$. Obviously such a value for the plume width is much too small to agree with observations.

\section{Summary and conclusions}

In this paper we studied the damping of shear Alfvén waves due to phase mixing in two-dimensional planar magnetic plasma equilibria. The equilibrium magnetic field was assumed to be potential. The only dissipative process taken into account was shear viscosity. We introduced curvilinear coordinates, which are the magnetic flux function and magnetic potential. When there is no viscosity, the equation describing the Alfvén wave propagation reduces to a one-dimensional wave equation with the variable phase speed (see Eq. (9)). This equation describes the Alfvén wave propagation along the magnetic field lines. The phase speed varies both along and across the field lines. Because of the phase speed variation across the field lines the Alfvén waves 
propagate with different speeds along neighbouring magnetic field lines meaning that these field lines oscillate more and more out of phase. This process is called phase mixing. As a result of phase mixing large gradients build up in the direction orthogonal to the magnetic field lines, which enormously enhances the Alfvén wave damping.

We then made the variable substitution that reduces the onedimensional wave equation describing the Alfvén wave propagation in the absence of viscosity to the Klein-Gordon equation (see Eq. (16)). We then imposed the condition that this equation has coefficients not varying along the magnetic field lines, which implies that the Alfvén waves propagate along the magnetic field lines without reflection. This condition results in the relation between the plasma density $\rho$ and the magnetic field magnitude $B$. It is given by either Eq. (23) or (24). It is interesting that in the case where $\rho$ does not change along the magnetic field lines, the Klein-Gordon equation becomes the wave equation with the coefficients not varying along the magnetic field lines. Of course, it is difficult to expect that the relation between $\rho$ and $B$ is exactly satisfied in the solar corona. However, we can expect that it is satisfied approximately. In this case there will be wave reflection, but only fairly weak. In particular, the assumption that the density does not vary along the magnetic field lines is approximately satisfied when the magnetic scale height is smaller than the density scale height. The analysis carried out by Smith et al. (2007) for short waves using the WKB approximation showed that the effect of density variation in the equilibrium with the exponentially divergent magnetic fields can be neglected when the density scale height is only two times larger than the magnetic scale height.

The Klein-Gordon with the coefficients not varying along the magnetic field lines and an additional term describing the viscous dissipation was studied in various equilibria. First we considered equilibia with the straight magnetic field lines. We started with the equilibrium where the density does not vary along the magnetic field lines and reproduced the result first obtained by Heyvaerts \& Priest (1983) that the wave amplitude is proportional to the exponent of the distance along the magnetic field lines cubed. Then we studied the wave damping in the case when the Alfvén speed increases either linearly or quadratically with the height. We found that the Alfvén wave increase decelerates the wave damping. In particular, in the case of the Alfvén speed quadratically depending on the height the wave amplitude tends to a constant non-zero limit at infinity.

We then proceeded to the wave damping in the equilibrium with the divergent magnetic field lines. Previously Ruderman et al. (1998) and Smith et al. (2007) considered this problem under the assumption that the wavelength is much smaller than the characteristic scale of the equilibrium quantity variation $H$. They found that the magnetic field divergence results in the enhanced Alfvén wave damping with the logarithm of the wave amplitude proportional to $-\exp (3 z / H)$. We obtained a similar result for waves with the wavelength comparable with $H$. We applied the obtained results to the damping of Alfvén waves propagating in coronal plumes. We found that the efficiency of damping depends on three dimensionless parameters: the ratio of the plume size at its base to the magnetic field scale height, $\xi_{b}$; the ratio of the densities at the plume centre to that far from the plume, $\varsigma+1$; and $\chi=\operatorname{Re} / \varpi^{2}$, where $\operatorname{Re}$ is the viscous Reynolds number and $\varpi$ the dimensionless wave frequency.
In our calculations we took $\varsigma=4, H=30 \mathrm{Mm}$, and $\xi_{b}=1 / 10$, which corresponds to the plume size at its base equal to $6 \mathrm{Mm}$. We found that for typical values of the magnetic field and plasma parameters, the shear viscosity $v \approx 1 \mathrm{~m}^{2} \mathrm{~s}^{-1}$. Our numerical results showed that to have waves with periods of the order of $60 \mathrm{~s}$ damped within 6 magnetic field scale heights, i.e. at a height approximately equal to $200 \mathrm{Mm}$, it is necessary to increase $v$ by about 6 orders of magnitude, i.e. to take $v \simeq 10^{6} \mathrm{~m}^{2} \mathrm{~s}^{-1}$. This increase can be caused by the plasma turbulence. It seems quite possible that the motion in an Alfvén wave undergoing phase mixing becomes turbulent because it is shear motion with the variation of the shear velocity on a very small spatial scale. Usually such a motion is subject to the KelvinHelmholtz instability. As concerns the estimate of the turbulent viscosity magnitude, it looks like a very difficult problem. The starting point in solving this problem should be the calculation of the instability threshold for oscillatory shear motion. To our knowledge this problem has not been solved yet.

Acknowledgements. The authors gratefully acknowledge financial support from the Russian Fund for Fundamental Research (RFFR) grant (16-02-00167). M.S.R. acknowledges the support from the STFC grant.

\section{References}

Bender, C. M., \& Orszag, S. A. 1999, Advanced Mathematical Methods for Scientists and Engineers (New York: McGraw-Hill)

Botha, G. J. J., Arber, T. D., Nakariakov, V. M., \& Keenan, F. P. 2000, A\&A, 363,1186

Braginskii, S. I. 1965, Rev. Plasma Phys., 1, 205

Cally, P. S. 2012, Sol. Phys., 280, 33

Didenkulova, I., Pelinovsky, E., \& Soomere, T. 2008, Proc. Est. Acad. Sci., Eng., 165,2249

De Moortel, I., Hood, A. W., Ireland, J., \& Arber, T. D. 1999, A\&A, 346, 641

De Moortel, I., Hood, A. W., \& Arber, T. D. 2000, A\&A, 354, 334

DeForest, C. E., Hoeksema, J. T., Gurman, J. B., et al. 1997, Sol. Phys., 175, 393 Ginzburg, V. L. 1970, Usp. Fiz. Nauk, 101, 185

Grimshaw, R., Pelinovsky, E., \& Talipova, T. 2010, J. Phys. Oceanogr., 40, 802 Heyvaerts, J., \& Priest, E. R. 1983, A\&A, 117, 220

Hood, A. W., Gonzalez-Delgado, D., \& Ireland, J. 1997a, A\&A, 324, 1114

Hood, A. W., Ireland, J., \& Priest, E. R. 1997b, A\&A, 318, 957

Hood, A. W., Brooks, S. J., \& Wright, A. N. 2002, Proc. R. Soc. Lond. A, 458, 2307

Hood, A. W., Brooks, S. J., \& Wright, A. N. 2005, Proc. R. Soc. Lond. A, 461, 237

Ibragimov, N. H., \& Rudenko, O. V. 2004, Acoust. Phys., 50, 406

Malara, F., Primavera, L., \& Veltri, P. 1996, ApJ, 459, 347

Nakariakov, V. M., Roberts, B., \& Murawski, K. 1997, Sol. Phys., 175, 93

Nakariakov, V. M., Roberts, B., \& Murawski, K. 1998, A\&A, 332, 795

Petrukhin, N. S., Pelinovsky, E. N., \& Batsyna, E. K. 2011, JETP Lett., 93, 564

Petrukhin, N. S., Pelinovsky, E. N., \& Talipova, T. G. 2012, Izv., Atmos. Ocean. Phys., 48, 169

Petrukhin, N. S., Ruderman, M. S., \& Pelinovsky, E. N. 2015, Sol. Phys., 290, 1323

Ruderman, M. S. 1999, ApJ, 521, 851

Ruderman, M. S., Nakariakov, V. M., \& Roberts, B. 1998, A\&A, 338, 1118

Ruderman, M. S., Goldstein, M. L., Roberts, D. A., Deane, A., \& Ofman, L. 1999, J. Geophys. Res., 104, 17057

Ruderman, M. S., Pelinovsky, E., Petrukhin, N. S., \& Talipova, T. 2013, Sol. Phys., 286, 417

Smith, P. G., Tsiklauri, D., \& Ruderman, M. S. 2007, A\&A, 475, 1111

Spitzer, L. 1962, Physics of Fully Ionized Gases (New York: Wiley Interscience)

Tsiklauri, D. 2016, A\&A, 586, A95

Tsiklauri, D., \& Nakariakov, V. M. 2002, A\&A, 393, 321

Tsiklauri, D., Arber, T. D., \& Nakariakov, V. M. 2001, A\&A, 379, 1098

Tsiklauri, D., Nakariakov, V. M., \& Arber, T. D. 2002, A\&A, 395, 285

Tsiklauri, D., Nakariakov, V. M., \& Rowlands, G. 2003, A\&A, 400, 1051 


\section{Appendix A: Evaluation of decrement in the short wavelength approximation}

We obtain the expression for the decrement in the equilibrium with exponentially diverging magnetic field lines under the condition that the wavelength is much smaller than the characteristic scale of the magnetic field variation $H$.

In Sect. 7 we assumed that the density is independent of $\phi$. However, for the comparison with the results obtained by Smith et al. (2007) it is more convenient to consider the density that is independent of $z$. In that case $\sigma \neq 0$. Let us compare the two terms on the right-hand side of Eq. (27). It follows from Eq. (15) that $\sigma /\left(\mu_{0} B_{0}^{2}\right)$ is of the order of $V_{\mathrm{A}}^{2} / H^{2}$. On the other hand, $\omega$ is much larger than $V_{0} / H$. Hence, the ratio of the second term to the first is much larger than $V_{\mathrm{A}}^{2} / V_{0}^{2} \sim 1$. This implies that the second term can be neglected and we can take $\lambda \approx \omega$.

Now we can no longer use Eq. (59), and must directly use Eq. (13) instead. Differentiating this equation with respect to $\psi$ we obtain

$\frac{\partial h}{\partial \psi}=B_{0} V_{0} \sqrt{\mu_{0}} \int_{\phi_{1}}^{\phi} \frac{\partial}{\partial \psi}\left(\frac{\sqrt{\rho}}{B^{2}}\right) \mathrm{d} \phi^{\prime}$.

Smith et al. (2007) assumed that the region where the wave propagates is narrow implying that $|x| \ll H$. Then it follows that $|\psi / \phi| \ll 1$. Using Eqs. (54) and (55) yields

$B^{2}=\frac{B_{0}^{2}}{H^{2}}\left(\phi^{2}+\psi^{2}\right)=B_{0}^{2} \mathrm{e}^{-2 z / H}$.

Now we obtain

$\frac{\partial}{\partial \psi}\left(\frac{\sqrt{\rho}}{B^{2}}\right)=\frac{1}{2 \sqrt{\rho} B^{2}}\left(\frac{\partial \rho}{\partial x} \frac{\partial x}{\partial \psi}-\frac{4 \rho \psi B_{0}^{2}}{H^{2} B^{2}}\right)$.

Using Eq. (54) we express $x$ in terms of $\phi$ and $\psi$, and then obtain with the aid of Eq. (A.2)

$\frac{\partial x}{\partial \psi}=-\frac{\phi B_{0}^{2}}{H B^{2}}$
Substituting Eqs. (A.2) and (A.4) in Eq. (A.3) yields

$\frac{\partial}{\partial \psi}\left(\frac{\sqrt{\rho}}{B^{2}}\right)=-\frac{\phi \sqrt{\rho} B_{0}^{2}}{2 H B^{4}}\left(\frac{1}{\rho} \frac{\partial \rho}{\partial x}+\frac{4 \psi}{H \phi}\right)$.

Since $|\psi / \phi| \ll 1$ we can neglect the second term in the brackets. In addition, since $|x| \ll H$ we can take $\phi \approx-H \mathrm{e}^{-z / H}$. Then, using these results and Eq. (A.2), we eventually obtain

$\frac{\partial}{\partial \psi}\left(\frac{\sqrt{\rho}}{B^{2}}\right)=\frac{\mathrm{e}^{3 z / H}}{2 \sqrt{\rho} B_{0}^{2}} \frac{\partial \rho}{\partial x}$.

It is convenient to make the variable substituting in the integral in Eq. (A.1) and integrate with respect to $z$ instead of $\phi$. When $\phi=\phi_{1}$ we have

$z=-\frac{H}{2} \ln \left(1+\frac{\psi^{2}-\psi_{b}^{2}}{H^{2}}\right) \approx 0$

Then, using $d \phi \approx e^{-z / H} d z$ and substituting Eq. (A.6) in Eq. (A.1) we obtain

$\frac{\partial h}{\partial \psi}=\frac{V_{0}}{2 V_{\mathrm{A} 0} \rho} \frac{\partial \rho}{\partial x} \int_{0}^{z} \mathrm{e}^{2 z^{\prime} / H} \mathrm{~d} z^{\prime}=\frac{V_{0}}{4 V_{\mathrm{A} 0} \rho} \frac{\partial \rho}{\partial x}\left(\mathrm{e}^{2 z / H}-1\right)$.

Substituting this result in Eq. (61) and using Eq. (62) we eventually arrive at

$\Lambda=\frac{v \omega^{2} H^{3}}{96 V_{\mathrm{A} 0}^{3} \rho^{2}}\left(\frac{\partial \rho}{\partial x}\right)^{2}\left(\mathrm{e}^{z / H}-1\right)^{3}\left(3 \mathrm{e}^{-z / H}+1\right)$.

This expression coincides with the expression for the decrement obtained by Smith et al. (2007) if we take $H_{\rho} \rightarrow \infty$ in the latter, which corresponds to the density independent of $z$. 